\title{
Imprimer les émotions : stratégies du narrateur dans la quatrième Histoire tragique de Bénigne Poissenot
}

\author{
To imprint emotions: narrator's strategy in the fourth \\ Histoire tragique by Bénigne Poissenot
}

\author{
Barbara Marczuk \\ Uniwersytet Jagielloński \\ marczukb@o2.pl
}

\begin{abstract}
Bénigne Poissenot was the first author to introduce the theory of tragic tales reception, which was a vastly popular genre by the end of 16th and the beginning of 17th century in France. In the prologue of Nouvelles Histories Tragiques (1586) he emphasizes that the reason of his tales is to move the readers deeply and make them choose to go through a moral transformation and to live virtuous lives. This article's objective is to investigate what methods and strategies the narrator uses to influence a reader's emotions and to what extent those emotions help (or not) deliver the moral message, which can be found in his first-person declarations (the introduction and the conclusion of the tale). It is done by examining the fourth novel.
\end{abstract}

Keywords: Renaissance tragic tales, rhetoric strategy, emotions in litterature

« Nous sommes ennuyés de livres qui enseignent, donnez nous-en pour émouvoir »

Agrippa d'Aubigné, avis Aux Lecteurs des Tragiques ${ }^{1}$

L'histoire tragique, genre acclimaté en France grâce à Pierre Boaistuau ${ }^{2}$ et jouissant d'une popularité immense jusqu'à la seconde moitié du XVII ${ }^{\mathrm{e}}$ siècle, constitue une des expressions littéraires « des passions déchaînées qui ont bouleversé la France

\footnotetext{
${ }^{1}$ Cité d'après Corbin \& al., 2016, p. 391.

${ }^{2}$ En 1559 il publie l'adaptation de six nouvelles de Matteo Bandello sous le titre Histoires Tragiques.
} 
des guerres civiles »(Carr, 1977, p. XLVII). Ces récits brefs, dont la matière était fournie par des faits divers et des chroniques criminelles, « représentaient au vif et exprimaient comme en un tableau » (Poissenot, 1996, p. 49) ${ }^{3}$ des actes violents et transgressifs : « concubignages, deflorements, adulteres, assassinats » (Poisssenot, 1996, p. 49) dans le but déclaré de prévenir le lecteur contre les désastres qui procèdent inéluctablement de tels crimes. Conçue dans le climat des passions violentes, l'histoire tragique cherche à « représenter les choses nument » (Poissenot, 1996, p. 49, je souligne) et à travers l'expression des émotions des protagonistes procurer au lecteur une violente perturbation d'esprit accompagnée d'un frisson d'épouvante ${ }^{4}$.

Bénigne Poissenot qui dans le Prologue de l'Auteur sur ses Histoires Tragiques évoque de manière élogieuse ses prédécesseurs (A.D.S.D., Pierre Boaistuau, François de Belleforest, Jacques Yver, Vérité Habanc), est le premier à proposer un bilan du genre à la mode et, ce qui est particulièrement important, à esquisser les lignes principales de la théorie de réception de ce type de récits ${ }^{5}$.

Dès l'incipit, Poissenot tâche d'expliquer le méchanisme psychologique qui permet de produire des émotions chez le lecteur par le biais de la narration habilement menée. Selon lui, « Ceux mesme qui n'endurent jamais facherie, quand ils viennent à considerer sans douleur les inconvenients d'autruy, sont esmeus de telle façon que la pitié qu'ils en ont ne peut qu'elle ne leur soit aucunement plaisante » $\left(1996\right.$, p. 45) ${ }^{6}$. Ce paradoxe de la «pitié plaisante», est largement orchestré dans la suite du Prologue et confirmé par l'autorité de Cicéron :

Les accidents et diverses fortunes d'un homme excellent et renommé remplissent le lecteur d'admiration, d'attente, de joie, de facherie, d'esperance, de crainte [...] Il n'y a chose plus apte et convenable, pour la delectation du lecteur, que la varieté des temps et les diverses mutations de fortune, lesquelles, combien qu'elles ayent esté de peu de plaisir à ceux qui les ont experimentées et souffertes, si est-ce qu'elles sont plaisantes et agréables à lire. (Poissenot, 1996, p. 46)

La raison du succès des histoires tragiques réside donc, selon Poissenot, dans cette aptitude à susciter des émotions extrêmes et par cela fournir au lecteur « le contentement d'esprit » (1996, p. 47). À la même époque des conceptions analogues sont

\footnotetext{
${ }^{3}$ Toutes les citations des Nouvelles Histoires tragiques (1586) d'après cette édition.

${ }^{4}$ Selon G. Vigarello, les textes du XVI ${ }^{\mathrm{e}}$ siècle s'orientent vers l'intériorisation du sens du mot «émotion » qui avant signifiait mouvement ou agitation corporelle (Corbin, 2016, p. 224). Au XVII ${ }^{\mathrm{e}}$ siècle, sous la plume de Descartes et de Furetière, les termes " émotion d'âme ", " émotion d'esprit " sont substituables à la passion (Corbin, 2016, p. 250 et 390). Dans la suite de l'article je me réfère à la distinction actuelle entre émotion (affect) - réaction affective intense mais de courte durée (crainte, pitié, joie, fâcherie, colère) et passion - état affectif intense et permanent (amour, haine, jalousie).

${ }^{5}$ Pour le commentaire judicieux de ce Prologue voir Pietrzak, 2006, p. 212.

${ }^{6}$ Cette explication correspond à l'idée d'Aristote : « Toutes les choses que nous craignons à notre sujet, émeuvent notre pitié quand elles arrivent à autrui » (1991, p. 83).
} 
formulées par les théoriciens français de l'ars dramatica: La Taille, dans sa lettretraité De l'Art de la tragédie (1573) répète, après Aristote, que le but principal du spectacle tragique consiste à éveiller la crainte et la pitié et par cela fournir aux spectateurs un plaisir esthétique :

La vraie et seule intention d'une Tragedie est d'esmouvoir et de poindre merveilleusement les affections d'un chascun car il faut que le subject en soit si pitoyable et poignant en soy, qu'estant mesmes en bref et nument dit, engendre en nous quelque passion. [...] Pleust à Dieu que les Roys et les grands sçeussent le plaisir que c'est de voir reciter et representer au vif une vraye Tragedie. (J. de La Taille, 1998, pp. 7 et 12, je souligne)

Les objectifs de l'histoire tragique ne se limitent pourtant pas aux seuls principes hédonistes de delectare et permovere. En divaguant sur le premier mot du terme générique : histoire, Poissenot souligne la supériorité de ce domaine de savoir sur la loi et la philosophie qui n'ont pas la même efficacité pour enseigner « à bien vivre, abhorrant le vice et suyvant le chemin de vertu» (1996, p. 55). Selon lui, la vigueur des lois est fondée sur la contrainte et la violence, la philosophie, en revanche, n'a aucun pouvoir d'entraver les péchés du monde. L'histoire, quant à elle, non seulement contient des exemples à suivre ou à éviter, mais

elle a avec cela des aiguillions, qui ne sont pas de peu de force, et qui peuvent convier, voire trainer malgré soy, les plus revesches, cruels et barbares, et qui se sont despouillez de toute humanité, en une manière de vivre toute autre que celle qu'ils gardoyent au paravant. (Poissenot, 1996, p. 55)

Ces constatations définissent de manière pertinente l'intention de l'auteur des Nouvelles Histoires tragiques : par le plaisir des émotions véhémentes il s'agit d'acheminer le lecteur, même à son insu et contre son gré, vers la pratique des vertus ${ }^{7}$.

Dans cette définition d'un discours efficace qui vise à influencer directement la volonté de l'auditeur, on peut déchiffrer en filigrane la leçon des rhéteurs latins, particulièrement de Cicéron, auteur évoqué explicitement au début du Prologue. Dans son traité De Oratore le philosophe explique la différence entre le sermo, locu-

\footnotetext{
${ }^{7}$ En soulignant le but moralisateur des histoires tragiques, Poissenot pare explicitement aux attaques de Cornelius Agrippa et Juan Luis Vivès contre les livres immoraux (cf. Prologue, p. 48). Par cette mise en avant de la valeur morale de la littérature, l'écrivain se rapproche des dramaturges contemporains qui, en interprétant la katharsis aristotelicienne, assignaient aux émotions de crainte et de pité la fonction édifiante : "La tragedia ha anco il suo diletto, e in quel pianto si scopre un nascoso piacere [...] che tragge gli animi alla attenzione [...] la quale gli fa bramosi di apparare, col mezzo dell'orrore e della compassione, quello che non sanno, cioè di fuggire il vizio e di seguir la virtù » (G. B. Giraldi Cinzio, Discorso intorno al comporre delle commedie e delle tragedie, cité d'après Maślanka-Soro, 2013, p. 161).
} 
tion des philosophes voulant instruire de manière objective et impassible et l'oratio visant à fléchir les esprits et séduire l'auditoire en exploitant le potentiel de l'affectivité (Mathieu-Castellani, 2001, p. 336). Cicéron souligne que la réception favorable du discours et l'acceptation des opinions qui y sont proclamées dépend moins de la justesse et véracité des arguments rationnels que des émotions que l'orateur est capable d'éveiller :

Les hommes forment leurs jugements beaucoup plus sous l'emprise de la haine ou de l'amour, de l'envie ou de la colère, de la douleur ou de la joie, de l'espoir ou de la crainte, de l'erreur ou de quelque émotion de leur âme [permotione mentis], que par respect de la vérité, de la jurisprudence, des règles juridiques, du droit ou des lois. (Cicéron, 1985, II, xlii, p. 178)

Selon Cicéron, pour gagner l'assentiment de l'auditoire, l'orateur lui-même doit être ému ou du moins sembler tel. Respectivement, dans un texte narratif, pour gouverner les émotions du lecteur il faut visualiser des passions intenses, exprimées par les protagonistes de manière pathétique.

La représentation des états affectifs dans les histoires tragiques a été largement étudiée par des chercheurs récents qui ont analysé la structure rhétorique de longues complaintes, le rôle des tirades, lettres, fragments poétiques (Pietrzak, 2006, pp. 257-301 ; Chupeau, 1981, pp. 225-227). Des travaux importants ont été aussi consacrés au langage du corps, amplement exploité dans ces récits qui se nourrissent des affects portés au paroxysme (Arnauld, 1995 ; Pietrzak, 2008, pp. 173-200). Moins d'attention a été prêtée au statut du narrateur dans les recueils et, plus particulièrement, aux procédés par lesquels il programme les émotions des lecteurs (Pietrzak, 2008, pp. 253-256). Les Nouvelles Histoires tragiques de Poissenot semblent à cet égard révélatrices. En premier lieu, en raison de la théorisation du problème (esquisse de la psychologie de réception entreprise par l'écrivain dans le Prologue déjà cité), mais aussi en conséquence de la conception du narrateur auquel Poissenot réserve un statut particulier dans l'économie du recueil.

Conformément au schéma figé du genre, le narrateur des Nouvelles Histoires tragiques s'exprime dans deux types de discours qui répondent à la définition cicéronienne. Dans chaque histoire, l'oratio affective (le récit à la troisième personne) est encadré par le sermo du narrateur-moraliste (argument, exorde, péroraison), mais la relation entre la portée émotionnelle de l'une et la doxa explicitée dans l'autre est souvent ambigüe sinon conflictuelle.

En analysant la quatrième histoire du recueil je me propose donc, dans le premier temps, de décéler les procédés narratifs utilisés dans l'oratio dans le but d'agir sur les affects de l'auditoire ; ensuite de sonder dans quelle mesure cet effet émotionnel peut corroborer la thèse morale détaillée dans le sermo du narrateur.

Le choix de cette nouvelle résulte de la place privilégiée que Poissenot lui a assignée dans son recueil. Située au centre, elle constitue le doublet de la célèbre 
première histoire Chastes amours de Floridanus et Eluide et la pitoyable fin de tous deux $x^{8}$. Les deux nouvelles illustrent l'affaire épineuse des mariages clandestins ${ }^{9}$ et réalisent le même schéma narratif : l'amour des jeunes contrarié par la famille, la fugue, le malheur qui en survient. Revenant deux fois dans le même recueil à cette question, l'écrivain met en lumière la prégnance du problème et fait vibrer la corde à laquelle son auditoire serait sans doute sensible.

Bénigne Poissenot, auteur effectif du recueil, se déclare explicitement comme narrateur des récits. Dans chaque exorde, il précise les circonstances dans lesquelles il a acquis la connaissance de ses histoires: les trois premières proviennent des sources écrites bien précises, les deux suivantes lui ont été racontées par une personne fiable, la dernière s'appuie sur ses souvenirs personnels. La quatrième histoire appartient à cette deuxième catégorie : elle a été confiée au jeune Poissenot (il déclare avoir eu à l'époque «dix-neuf à XX ans ») par un « honneste homme se disant estre du duché de Bourgogne » (Poissenot, 1996, p. 170) qu'il a rencontré à Aix en $1577^{10}$. Ces précisions autobiographiques non seulement assurent la crédibilité du récit, mais permettent aussi au narrateur de nouer une relation intime avec « l'ami lecteur » (Poissenot, 1996, p. 62) et de créer un climat de confidence. De surcroît, en dévoilant ses sources, Poissenot renonce au statut démiurgique du narrateur omniscient, créateur et maître de la diégèse, pour prendre la position d'un truchement, intermédiaire entre l'histoire (dont il est le premier auditeur) et le public de son livre ${ }^{11}$.

Le désir de susciter des émotions et par cela influencer le jugement moral du lecteur est perceptible dès le titre de la nouvelle. Temeraire entreprise du clerc d'un Conseiller d'Aix en Provence qui, après avoir abusé de la fille de son Maistre, la voulut enlever : et comme le Conseiller, en estant averti, y remedia discrettement. Ce long intitulé annonce un récit de transgression de l'ordre familial dans lequel le jeune homme sera fautif, la fille victime de «l'abus » et le père punisseur judicieux.

La mise en place des dramatis personae ne provoque pas de sympathie excessive : ils proviennent du milieu banal de la bourgeoisie de robe, sont privés de noms propres (même fictifs), ce qui leur ôte l'individualité, et leur présentation se limite aux traits typiques de leur âge et de leur état : la fille est « preste à marier »,

\footnotetext{
${ }^{8}$ Cette nouvelle a été l'objet de nombreuses études répertoriées par Suard, 1990 ; cf. aussi Marczuk, 1997.

${ }^{9}$ Dans l'argument de la première histoire, Poissenot évoque l'ordonnance du roi Henri II de 1556 obligeant les parents à déshériter les filles de moins de vingt-cinq ans et des garçons de moins de trente ans qui se sont mariés sans leur consentement (Poissenot, 1996, p. 67, note 4).

${ }^{10}$ Poissenot ne précise pas cette date, mais le meurtre du comte de Montafier que son interlocuteur évoque a été commis cette année-là.

${ }^{11}$ Avec une cartaine nonchalance Poissenot signale que son savoir concernant les détails de l'affaire n'est pas complet: « Nous ne nous informasmes point par le menu de celuy duquel tenons la presente histoire de ceste particularité... » (1996, p. 197).
} 
le clerc du Conseiller, "attentif au service de son maistre », apprend un métier qui n'est pas des plus honnêtes (il consiste « à tondre la brebis et à plumer l'oye sans la faire crier », Poissenot, 1996, p. 179).

Dès cette «préparation du lieu », le narrateur maintient une certaine distance par rapport à la diégèse qui devra illustrer la thèse largement exposée dans l'argument : l'impératif de loyauté des subalternes envers leurs maîtres, surtout dans le domaine de la morale domestique. Le recul du narrateur est mis en évidence aussi par le fait qu'il n'entre pas tout de suite dans le vif du sujet. Il tient le lecteur en suspens en dialoguant avec lui sur six pages (dans l'édition moderne) au sujet du bienfait des voyages, avant de faire sortir le protagoniste principal comme un fantoche d'une boîte à marionnettes : « Il y a trop longtemps que notre Clerc dort, esveillons-le pour le faire entrer au service du Conseiller » (Poissenot, 1996, p. 176).

L'attitude condescendante envers les protagonistes caractérise toute la première partie du récit qui se déroule selon le schéma de la nouvelle sentimentale ${ }^{12}$. Conformément à la convention de ce genre le narrateur laisse une large place au discours direct: les étapes de l'aventure affective sont jalonnées par complaintes, délibérations, tirades lyriques et stratégiques, cadeaux en forme de sonnets. Loin d'être des ornements rhétoriques superflus, ces discours permettent de donner la vie aux personnages (Mandrou, 1961, p. 74), de fournir des éléments de justification psychologique et de faire ressortir le drame de la vie intérieure (Carr, 1977, p. LXIX). Chaque discours du protagoniste est préparé par des symptômes corporels spontanés et incontrôlables qui, en premier lieu, témoignent de l'intensité du trouble émotif, mais qui aussi doivent produire un effet violent sur le lecteur ${ }^{13}$ :

Elle demeura assez bonne espace de temps sans luy faire autre response que d'un fleuve de larmes, duquel sa face estoit du tout arrosée. Puys, sanglottant et souspirant aussi fort que le coeur eust deu luy saillir hors du ventre, elle luy respondit ce qui s'ensuit... (Poissenot, 1996, p. 194)

Conformément au protocole du récit sentimental, le lecteur devrait s'attendrir sur le spectacle du corps souffrant et de l'âme en peine, compatir avec les héros et espérer l'aboutissement favorable de leur amour.

\footnotetext{
${ }^{12}$ R. Carr dans son Introduction aux Histoires tragiques de Pierre Boaistuau a établi la différence, devenue classique, entre la nouvelle sentimentale le récit tragique. La première, plus longue, cherche à inspirer la crainte et la pitié ; la seconde, concise, doit provoquer le frisson d'épouvante (Carr, 1977, pp. LXVIII-LXIX).

${ }^{13}$ En accord avec la recommandation Horatienne : «Car la nature nous modèle d'abord au-dedans selon tous les états divers de la fortune, elle nous inspire la joie, nous pousse à la colère, ou bien nous plie jusqu'à terre sous le poids du chagrin et nous serre le cœur; puis elle révèle au dehors les mouvements de l'âme en prenant la langue pour interprète » (Horace, 1995, vv. 108-111). Sur la relation entre l'expression corporelle et verbale des émotions, voir les considérations importantes de DimkeKamola, 2014.
} 
Néanmoins, l'attitude du narrateur empêche ce type de lecture. Par des formules ironiques : « monsieur le Clerc », « nostre amoureuse » (Poissenot, 1996, p. 191), il marque une distance par rapport à leur aventure sentimentale et impose la même attitude au lecteur. Pareil effet est produit par les digressions moqueuses, comme par exemple le passus à propos de la vanité des filles « qui suporteront plus patiemment d'estre appellées larronnesses, maraudes et injuriées de toute autre sorte que d'endurer d'estre blamées de laideur » (Poissenot, 1996, p. 185). En plus, certaines effusions lyriques des protagonistes ne se prêtent pas à une lecture confiante. L'accumulation d'allusions intertextuelles dans les complaintes ${ }^{14}$ ou la construction en écho des tirades des amoureux dénoncent trop le caractère de pastiche littéraire pour pouvoir fléchir les émotions du lecteur. Le premier soliloque du Clerc en fournit un exemple spectaculaire : des topoï pétrarquistes « je meurs, je brusle » se combinent avec la phraséologie platonicienne «Je ne vis plus en moy, mais en la chose que j'ayme le plus en ce monde » pour déboucher, en fin de compte, sur des métaphores militaires relevant du registre gaillard « ma dame a de si forts et beaux terriers que de là-sus elle peut battre et canonner mes bastions les mieux munis et remparez » (Poissenot, 1996, pp. 180-181). La tirade de refus, prononcée par la fille, exploite en revanche des images de l'oisellerie, empruntées au fonds des proverbes : «Sachez que depuis qu'un a une fois descouvert les rets du chasseur, il a beau abattre les buissons, car les oiseaux ne s'iront pas jetter dedans » (Poissenot, 1996, p. 182) ${ }^{15}$. L'ornementation rhétorique ostentatoire constitue un clin d'œil de la part du narrateur et entraîne le lecteur dans une sorte de connivence intellectuelle, au lieu de l'émouvoir.

La stratégie qui doit contrevenir à l'engagement affectif du lecteur atteint son apogée au moment de la «conjonction » des amoureux, acte qui ne devrait pas être accompli dans un récit sentimental décent. La chose même est relatée par le narrateur sur un ton moqueur et volontairement grivois, pointée par un jeu de mots égrillard :

Le Clerc visita si souvent le parvis de sa dame que ses coups ne furent semblables au passage de la couleuvre pardessus la pierre, et à la voie du navire trenchant les ondes marines sans que trace aucune apparoisse de son passage. Il avoit appris au service de son maistre à grossoyer les minutes [...] il apprint aussi à grossoyer les filles, et bastit si bien sur le devant de la maison de son amoureuse que le ventre luy enfla. (Poissenot, 1996, p. 191)

\footnotetext{
${ }^{14}$ Par exemple la phrase du Clerc « Si d'un hiver elle veut combattre mon Esté » (Poissenot, 1996, p. 181) : le dernier mot fait allusion à L'Esté : une autre œuvre de Poissenot (1583). Cf. aussi les paroles du narrateur: « ceux qui esprouvent les avantures estranges du monde adventureux » (p. 199) qui évoquent le recueil de A.D.S.D. Les Comptes $d u$ Monde adventureux (1555), cité aussi dans la péroraison de la première histoire (Poissenot, 1996, p. 118).

${ }^{15}$ Le même dicton est évoqué dans la première histoire, d'abord par le narrateur (Poissenot, 1996, p. 79), puis par Eluide dans sa tirade (p. 83), ce qui témoigne d'un jeu intertextuel délibéré.
} 
Pastiche littéraire, ironie, ton enjoué et licencieux font basculer le récit sentimental vers la nouvelle plaisante et, conformément au protocole propre à ce genre, imposent au lecteur une distance par rapport aux personnages. Par la peinture rabaissante et grivoise de l'amour, le narrateur veut empêcher le lecteur de devenir complice d'une transgression flagrante. Il faut souligner néanmoins qu'en désaccord avec la tendance majeure des récits tragiques, le conteur ne formule pas de réprobation de l'amour illicite. Il présente le délit perpétré par les amoureux sous un jour facétieux, amuse le lecteur au lieu de lui faire ressentir l'horreur du péché (ce dernier mot, d'ailleurs, n'apparaît pas dans cette nouvelle).

La stratégie narrative change au moment où l'histoire commence à prendre un tour dramatique ${ }^{16}$. Le narrateur s'estompe, tâchant de montrer les événements de manière neutre. Les soliloques de la fille qui se rend compte de sa grossesse, ainsi que les discours du Clerc qui essaie de remédier au désastre, ont un caractère plus dépouillé, le plaisir raffiné du beau langage cède la place aux résolutions morales : «Monstrez-vous d'un courage viril, et foullons nostre malheur aux pieds. La fortune a de coustume d'aider les courageux et de repousser les couards » (paroles du Clerc : Poissenot, 1996, p. 195).

Les protagonistes, malmenés par le conteur dans la partie sentimentale de l'histoire, commencent à susciter la compassion du lecteur et la crainte pour leur sort. Si le narrateur intervient à la première personne, ce n'est pas pour ironiser, mais pour débiter une prémonition cassandrique d'un malheur imminent :

L'esprit de l'homme, par je ne scay quelle force occulte, presage ordinairement ce qui est à advenir, comme il apparut en cestuy cy [le Clerc], qui estoit tant triste et melencholique que qui eust pris garde à sa face et contenance aisement eust jugé [...] qu'il attendoit partie de ce qui luy arriva. (Poissenot, 1996, p. 198)

La mort du jeune homme est relatée de manière sèche et objective, sans aucun détail naturaliste ni le moindre indice des sentiments et souffrances éprouvés par la victime $^{17}$. Les « rudes villains » (Poissenot, 1996, p. 199), engagés par le Conseiller, accomplissent l'assassinat de manière mécanique et froide, comme un impératif de justice. Cette mise en scène implacable suggère qu'il ne s'agit pas d'un meurtre, mais d'une exécution légitime, faite sur la personne d'un malfaiteur. Une fois le Clerc « jetté dedans la rivière » avec « une pesante pierre au col », le narrateur prend la position de moraliste pour formuler une leçon irrécusable :

${ }^{16}$ E. Boggio-Quallio souligne que Poissenot, de même que Jacques Yver, ne tendent pas à créer une atmosphère tragique depuis le début de leurs récits. Leurs histoires sont de « belles fables sentimentales qui se terminent par une conclusion qui inspire la pitié » (1981, p. 215).

${ }^{17} \mathrm{Ce}$ respect du decorum, caractéristique de la représentation de la mort par Poissenot, le distingue des auteurs des décennies suivantes. Jean-Pierre Camus et François de Rosset, sacrifiant au goût de la delectatio morosa, aimeront étaler devant les yeux de l'auditoire des « spectacles d'horreur » représentés dans un « amphithéâtre sanglant ». 
Sur luy prendront exemple tous serviteurs domestiques pour ne s'oublier sy avant en leur devoir que d'oser offenser leur maistre [...] paillardant avec sa femme, fille ou autre de sa maison, à laquelle ils sont tenus de porter autre respect que d'en faire le bordeau. (Poissenot, 1996, p. 199)

La justesse de la punition et son caractère inéluctable doivent susciter l'effroi du lecteur, conformément à la pédagogie de la peur, seule efficace dans le domaine des transgressions affectives. Là où la persuasion rationnelle est vaine, il faut avoir recours aux émotions extrêmes qui, selon la formule du Prologue, pourront donner au lecteur des « aiguillions » pour changer la «manière de vivre ».

La structure figée de l'histoire tragique : loi - transgression - punition (Poli, 1991, p. 7) imposerait au narrateur de clôre son récit au moment où le coupable est puni et la leçon prononcée, d'autant plus qu'elle cadre avec la thèse libellée par le Bourguignon à l'usage du jeune Poissenot dans l'exorde : « Tout homme devait s'abstenir de deux vices, ennemis capitaux d'honnesteté [...] sçavoir est de la paillardise et du jeu » (Poissenot, 1996, p. 171). Néanmoins, la narration ne s'arrête pas là. À partir du passage de la nouvelle sentimentale au récit dramatique le narrateur met en exergue le personnage de la demoiselle qui deviendra une des créatures les plus attachantes de son recueil. Il faut rappeler que ni dans l'argument ni dans l'exorde, il n'a assigné à la fille du Conseiller aucune thèse à illustrer. De ce fait l'héroïne non seulement jouit d'une autonomie que le Clerc n'avait pas, mais acquiert aussi une profondeur morale qui lui fait gagner la sympathie et la compréhension du lecteur. Son aventure existentielle, dont on peut suivre la progression grâce aux soliloques, constitue un roman initiatique pénétrant.

Dès le début de l'histoire, sa part de responsabilité dans le délit est montrée comme moindre que celle du Clerc. Plusieurs fois elle s'appelle elle-même « chetifve et infortunée » (Poissenot, 1996, p. 193) soulignant sa position de faible victime. L'initiative amoureuse vient du jeune homme, la demoiselle « quelque resistence qu'elle fist, s'excusant sur les dures loix de son honneur » se laisse finalement emporter par la tourmente, presque à son insu, sans avoir formulé dans son cœur une acceptation consciente et volontaire. La constatation du narrateur qu' « enfin il [le Clerc] la rangea à son vouloir » (Poissenot, 1996, p. 190) scelle définitivement la faute de celui-ci ${ }^{18}$.

Dès le moment où la fille découvre sa grossesse, le narrateur renonce à tout commentaire à son sujet. En revanche, il étale devant les yeux du lecteur le spectacle pitoyable des troubles physiques, causés aussi bien par son état (« les esvanouissements, foiblesses et degoust de viandes », Poissenot, 1996, p. 191) que par des émotions excessives, plus pénétrantes que les souffrances d'amour, chantées de manière poétique dans la première partie : «L'apprehension de tant de dangers faisoit trem-

\footnotetext{
${ }^{18}$ Le narrateur semble plus compréhensif envers les filles qui sont « d'une nature plus tendre » (Poissenot, 1996, p. 177), ce qui permet de chercher à les disculper.
} 
bler de peur tout le corps de ceste jeune fille, lui faisoit dresser les cheveux en teste, et luy arrestoit tout court la parolle en la bouche » (Poissenot, 1996, p. 196).

L'effet de l'hypotypose du corpus dolens est intensifié, en accord avec la poétique humaniste, par des similitudes mythologiques : «Ceux qui pour avoir veu le chef de Meduse [...] n'estoient point plus esbahis que ceste dolente fille se trouva esperdue, entendant la mort de son amy »(Poissenot, 1996, p. 200).

La visualisation des troubles corporels exerce un effet puissant sur l'imagination du lecteur et éveille la compassion pour la fille. L'intensité de la relation émotionelle avec la demoiselle est corroborée par la teneur des soliloques qui permettent de suivre son mûrissement moral. Après le constat spontané de la responsabilité de son amant, elle élude généreusement cette pensée et reconnaît la faute comme sienne : « Je ne puis toutefois m'absoudre qu'il n'y ait de la coulpe de mon costé, et que je n'ay grandement failly, ne le refusant rigoureusement, ainsi que les loix de mon honneur me le commandoient » (Poissenot, 1996, p. 193).

Son sentiment de culpabilité évolue et devient encore plus poignant lorsqu'elle apprend l'assassinat de son ami. Dans la tirade adressée à son père, elle prend généreusement sur soi la responsabilité entière du forfait :

C'estoit donc à moy que deviez vous adresser [...] et non à cest Innocent qui, sans avoir offencé qu'à mon instigation, a neantmoins porté la paste au four pour l'amour de moy. [...] Qui devoit recevoir la punition qu'il a recue, sinon moy qui avois faict un tour de femme legere et trop mal prevoyante, lors que je luy permy de cueillir la premiere fleur de ma virginité. (Poissenot, 1996, pp. 200-201)

La lucidité, dont ces discours témoignent, constitue le point d'aboutissement de l'itinerarium mentis de la protagoniste. La conscience de la faute, le repentir, le sentiment de honte, l'acceptation héroïque de la responsabilité, par delà la tentation du suicide, restituent sa dignité. En plus, arrivée au bout de son périple moral, elle découvre, à la manière des héros tragiques, le principe qui détermine et gère le destin humain. Non par hasard dans ses locutions, de même que dans celles de son ami, « Dame Fortune », « ingratte, aveugle [...] legere et sans racine » (Poissenot, 1996, p. 187) est évoquée et invoquée plusieurs fois. C'est elle, «constante en ses mobilités » qui est reconnue par les héros (mais aussi par le narrateur) comme l'unique transcendance $^{19}:$ « Je dois bien cognoistre maintenant tes mobilitez, trompeuse et espouventable fortune qui te dis esperance des miserables et cruelle misere des prosperans » (paroles de la fille : Poissenot, 1996, p. 202). Le monde est non seulement «adventureux » (Poissenot, 1996, p. 199) mais surtout irrationnel et inexplicable. La prise de conscience de cet état des choses clôt le dernier soliloque de la demoiselle.

${ }^{19}$ Selon les critiques, les considérations sur le règne de Fortune constituent le principe organisateur du recueil (Préface de J. C. Arnould, R. Carr in Poissenot, 1996, p. 7). En revanche, les apostrophes à Dieu qui apparaissent ça et là, ne sont que des exclamations convenues. 
Le conteur non seulement affine le portrait moral de son héroïne, mais il imagine aussi pour elle un meilleur sort que celui du Clerc. Au lieu de la faire mourir, il lui permet d' « essuyer les larmes et se consoler avec le temps, qui seul est suffisant de mitiguer les plus grandes destresses et fascheries » (Poissenot, 1996, p. 204). De cette manière, le conteur satisfait l'attente du public dont il a soigneusement programmé la sympathie pour la fille au fil de la narration.

A la différence de son ami, transgresseur judicieusement puni, la demoiselle semble avoir échappé à l'intention univoquement didactique du récit, libellée dans l'argument et l'exorde. La conception de ce personnage génère une discordance entre le but moralisateur, affiché dans le sermo du narrateur, et l'effet rhétorique obtenu par l'oratio émotive. L'inconséquence encore plus flagrante concerne le personnage du Conseiller à qui, dans l'argument, le titre et l'exorde, le narrateur a assigné la position du justicier, garant de l'ordre moral. Néanmoins, son entrée en scène est non seulement brusque mais aussi dépeinte à l'aide de métaphores zoologiques dépréciatives : « Les yeux [...] luy roulloient dedans la teste, et mordant ses doigts [...] il groumelait entre ses dents et disait Patenostre du singe (Poissenot, 1996, p. 197) » ${ }^{20}$.

La violence verbale qu'il exerce ensuite sur sa fille est aussi rude qu'injuste et produit un effet de contraste violent avec la contrition et la résignation généreuse de celle-là. La vitupération qu'il émet est émaillée par des invectives vulgaires, épithètes dénigrantes et métaphores hyperboliques : « ma dame la putain », « ribaudes chaleurs », « effrenée paillardise », « insatiable volupté ». L'enfant conçu est appelé « germe adulterin », « l'oeuf d'un si meschant corbeau qui ne merite pas d'esclore sur terre » (Poissenot, 1996, p. 200).

Cette rhétorique de l'excès renforce la solidarité du lecteur avec la fille humiliée, au lieu de gagner son assentiment au réquisitoire du père. Le garant de l'ordre moral se trouve compromis par son propre discours. De ce fait, la stratégie d'intimidation qui devrait susciter la honte des lectrices (susceptibles de s'engluer dans une situation pareille), manque son but.

Le désaccord entre la leçon explicite et la portée émotionnelle du récit ne permet pas de le considérer comme l'illustration d'une thèse relevant de la morale pratique. Conformément aux intentions détaillées dans le Prologue, Poissenot a « représenté au vif et exprimé comme en un tableau » (1996, p. 49) les troubles psychiques, moraux et physiques, étant certes, la conséquence inéluctable des infractions à l'ordre patriarcal de la société ${ }^{21}$, mais qui surtout démontrent « dru et menu » «les

${ }^{20}$ Dans la Première histoire les métaphores zoomorphes: magots, matins, boucs lascifs, tigre (Poissenot, 1996, p. 116-117) étaient réservées aux «barbares rustiques » qui voulaient déflorer Eluide, donc aux personnages qui indiscutablement incarnaient le mal.

${ }^{21}$ Il est surprenant que Poissenot, ligueur et catholique fervent (Arnould, 1993), n'évalue pas les amours avant le mariage à la lumière du sixième commandement. Contrairement au protocole habituel des histoires tragiques, ni le narrateur ni les protagonistes ne parlent de la transgression de la loi divine. 
subites mutations » de la Fortune. Ceux qui avec témérité veulent la défier et lui « faire la figue » comme dit le Clerc (Poissenot, 1996, p. 196), seront immanquablement confondus et désillusionnés. Le narrateur a fait passer cette vérité en programmant les émotions du lecteur : il a capté la bienveillance pour lui-même, gagné la sympathie pour les protagonistes, suscité la crainte pour leur sort et la compassion aux moments pathétiques. Contrairement au modèle lancé par Boaistuau et Belleforest, il n'a pas insisté sur l'abomination de la transgression, mais il a tâché de mettre en avant le caractère inéluctable et cruel de la punition (physique dans le cas du Clerc et morale dans le cas de la fille). Tout en limitant ses interventions dans la partie dramatique du récit, il a réussi à imprimer les émotions «d'attente, de joie, de facherie, d'esperance, de crainte » (Poissenot, 1996, p. 46) subrepticement, laissant le lecteur face-à-face avec l'evidentia du corps souffrant, l'efflorescence rhétorique des discours et le pathos des tableaux de violence.

La quatrième histoire, de par ses potentialités esthétiques et émotives, avait de quoi délecter et émouvoir le lecteur. On peut douter, en revanche, qu'elle fût à même de lui donner des « aiguillions » pour suivre le chemin de la vertu. Elle a dépeint, certes, un tableau troublant du monde tel qu'il va, mais la discordance entre l'instruction contenue dans le sermo et la séduction exercée par l'oratio invitaient le lecteur à formuler son propre jugement: «Nous te faisons juge du fait raconté en l'histoire suivante [...] après l'avoir leue, tu diras ton advis si bon te semble » (Poissenot, 1996, p. 235).

Ce doute inquiétant qui s'insinue entre la leçon déclarée par le narrateur et l'effet exercé sur les émotions du lecteur, caractéristique des histoires tragiques en général, a certainement contribué au succès de ce genre auprès du public jusqu'au milieu du XVII ${ }^{\mathrm{e}}$ siècle $^{22}$.

\footnotetext{
${ }^{22}$ Le dernier recueil de ce type : Histoires tragiques de nostre temps de J.-N. Parival, parait en 1656.
} 


\section{BIBLIOGRAPHIE}

Aristote (1991). Rhétorique (M. Dufour, Trans.). Paris : Belles Lettres.

Arnould, J. C. (1993). Sur la sixième des « Nouvelles histoires tragiques » de Bénigne Poissenot. In P. Salwa, D. Żółkiewska (Eds.), Narrations brèves. Mélanges de littérature ancienne offerts à Krystyna Kasprzyk (pp. 205-215). Warszawa : Publications de l'Institut de Philologie Romane de l'Université de Varsovie.

Arnould, J. C. (1995). Les visages de la douleur dans les récits tragiques du XVI siècle. In B. Yon (Ed.), La Peinture des passions de la Renaissance à l'âge classique (pp. 49-59). SaintEtienne : Publications de l'Université de Saint-Etienne.

Boggio-Quallio, E. (1981). La structure de la nouvelle tragique de Jacques Yver à Jean-Pierre Camus. In J. Lafond, A. Stegmann (Eds.), L'Automne de la Renaissance 1580-1630. Actes du XXII Colloque International de Tours, 2-13 juillet 1979 (pp. 209-217). Paris : Vrin.

Carr, R. (1977). Introduction. In P. Boaistuau, Histoires Tragiques (pp. IX-LXXXIV). Genève : Droz.

Chupeau, J. (1981). Quelques formes caractéristiques de l'écriture romanesque à la fin du XVI siècle et au début du XVII ${ }^{\mathrm{e}}$. In J. Lafond, A. Stegmann (Eds.), L'Automne de la Renaissance 1580-1630. Actes du XXII Colloque International de Tours, 2-13 juillet 1979 (pp. 219-230). Paris : Vrin.

Cicéron (1985). De l'Orateur (E. Courbaud, Trans.). Paris : Belles Lettres.

Corbin, A., Courtine, J.-J., Vigarello, G. (Eds.) (2016). Histoire des émotions, vol. I : De l'antiquité aux Lumières (G. Vigarello, Ed.). Paris : Seuil.

Dimke-Kamola, J. (2014). Czytanie ciała. Problem werbalnej i niewerbalnej ekspresji emocji w piśmiennictwie włoskiego Renesansu. In A. Rzepka, D. Pudo, M. Wrana (Eds.), Pismo, lektura, biblioteka $w$ dawnych literaturach romańskich (pp. 133-145). Kraków : Collegium Columbinum.

Horace (1995). Art Poétique. In Horace, Epîtres (F. Villeneuve, Trans.). Paris : Belles Lettres.

La Taille J. de (1998). De l'Art de la Tragédie. In J. de La Taille, Saul le Furieux, La Famine ou les Gabéonites (G. Spillebout, Ed.). Mont-de-Marsan : Éditions José Feijóo.

Mandrou, R. (1961). Introduction à la France moderne. Paris : Albin Michel.

Marczuk, B. (1997). Floridan et Elvide, cinq versions du récit reconsidérées. In A. Bartosz, K. Dybeł, P. Tylus (Eds.), Jeux de la variante. Mélanges offerts à Anna Drzewicka (pp. 54-66). Kraków : Viridis.

Maślanka-Soro, M. (2013). Powrót Melpomeny. Tragedia wtoska od średniowiecznego odrodzenia po renesansowy rozkwit. Kraków : Księgarnia Akademicka.

Mathieu-Castellani, G. (2001). Le mécanicien, l'acteur et le charmeur de serpents : la rhétorique et les passions. In F. Lecercle, S. Perrier (Eds.), La Poétique des passions à la Renaissance (pp. 333-351). Paris : Champion.

Pietrzak, W. K. (2006). Le Tragique dans les nouvelles exemplaires en France au XVe siècle. Łódź : Wydawnictwo Uniwersytetu Łódzkiego.

Poissenot, B. (1996). Nouvelles Histoires tragiques [1586] (J. C. Arnould, R. Carr, Eds.). Genève : Droz.

Poli, S. (1991). Histoire(s) Tragique(s). Anthologie/Typologie d'un genre littéraire. Fasano Paris : Schena-Nizet.

Suard, F. (1990). Floridan et Elvide aux XV et XVI ${ }^{\mathrm{e}}$ siècles. In B. Alluin, F. Suard (Eds.), La Nouvelle. Définitions, transformations (pp. 163-179). Lille : Presses Universitaires de Lille. 